\title{
Réflexions éthiques critiques sur les directives de l'ASSM
}

\section{François Gachoud}

Philosophe, Bulle

\author{
Dans ses directives médico-éthiques sur "L'attitude face à la fin de vie et à la mort», \\ l'Académie Suisse des Sciences Médicales (ASSM) fait des propositions hautement \\ discutables. Promulguées en mai 2018, ces directives seront soumises à la fédéra- \\ tion faîtière des médecins suisses (FMH) qui décidera celles qui seront retenues \\ ou non. Je note pour ma part que, selon sondage, la majorité du personnel soignant \\ est favorable à ces directives. Je propose ici un examen éthique critique de ces di- \\ rectives.
}

Ce qui traverse de bout en bout le texte des directives, c'est la remise en cause radicale de la reconnaissance d'un critère objectif jugé nécessaire pour fonder l'autorisation de recourir au suicide assisté. Elle le remplace par le seul critère de la subjectivité du patient en souffrance. Avec de lourdes conséquences qui en découlent.

\section{Explications}

En principe et jusqu'ici, le choix de se donner la mort, avec une assistance possible selon l'art. 115 du Code pénal, était réservé aux seuls patients qui sont en fin de vie et qui souffrent d'une maladie incurable. Ce double critère garantissait l'objectivité fondant la pratique de l'assistance au suicide: la maladie est incurable et le patient est en fin de vie. Cette pratique relevait de l'exercice d'une expertise médicale. Le médecin posait des garde-fous qui ne sont pas requis par la loi mais par les codes de déontologie: la fin de vie est proche, la personne souffre d'une maladie mortelle et on explorait d'autres pistes pour pallier la souffrance de la personne malade. Mais depuis que les Associations Exit et Dignitas ont réussi à élargir l'assistance au suicide aux «polypathologies invalidantes», le patient n'est plus nécessairement en fin de vie et sa maladie n'est pas nécessairement incurable. L'ASSM emboîte clairement le pas et va même plus loin. Elle élargit le champ d'application «aux patients qui souhaitent une aide médicale pour mettre fin à leur vie, que la mort soit imminente ou non». Elle ajoute: «le patient demande de pouvoir choisir le moment de sa propre mort» (ch. 1 Champ d'application). Et elle précise qu'il s'agit bien de "patients dont le désir de suicide n'est pas motivé par une maladie» (ibidem).

Le seul motif alors avancé est celui de l'autodétermination du patient qui juge sa souffrance insupportable, quel que soit son âge. Ce qui justifie la demande de suicide assisté est donc une référence au seul sentiment subjectif du patient moyennant sa capacité de discernement. Ne prendre en compte que l'autonomie du patient et sa souffrance subjective, c'est pourtant prendre le risque de méconnaître le caractère souvent équivoque de la demande d'assistance au suicide. Mais l'ASSM n'hésite pas à privilégier ce seul critère: «Il n'existe aucun critère objectif pour évaluer la souffrance en général, ni le degré de souffrance supportable» (ch. 2.2.4).

A partir de là, et selon cette logique, n'importe qui et quel que soit son âge peut revendiquer le droit au suicide assisté sur la base de sa seule estimation personnelle. L'ASSM exclut clairement la nécessité de se référer à un critère objectif. Mais ce n'est pas parce qu'une souffrance est toujours en effet vécue subjectivement qu'on doit en déduire l'exclusion d'un critère objectif définissable. Ce critère a été défini jusqu'ici par une situation et un état objectivement constatables: le patient est vraiment en fin de vie, sa mort est réellement imminente et sa maladie est incurable. 


\section{Un fâcheux laxisme}

L'ASSM change donc fondamentalement la donne. Il suffit au fond de dire: «Je me déclare fatigué de la vie et je suis capable de discernement. Donc je puis m'autodéterminer à choisir le suicide quand je veux puisque je trouve ma souffrance insupportable.» L'ASSM cautionne en fait cette démarche purement subjective. Elle propose certes à l'appui un principe éthique reconnu important: celui du droit à l'autodétermination du patient jugé capable de discernement. Mais en l'occurrence, il ne s'agit pas de nier la validité de ce principe. Il s'agit de considérer toute la gravité de son application. Car c'est ici la vie elle-même qui est remise en cause: le patient veut se donner la mort!

L'ASSM prend ainsi le risque de mettre les médecins dans une situation délicate, même difficile, car comment mesurer et évaluer une souffrance subjective déclarée insupportable, surtout s'il ne s'agit pas d'une maladie incurable ou dans le cas de personnes qui ne sont pas malades mais demandent assistance au suicide ou encore dans le cas d'adolescents en détresse pour des raisons qui, à cet âge, traduisent une fragilité évidente et des retournements de décisions courantes. Malgré toutes les précautions prévues par l'ASSM dans ses directives, il sera difficile, voire impossible, d'évaluer la situation de ces cas. Le critère de l'objectivité jusque-là admise, soit la fin de vie et la maladie incurable, offre des garanties bien plus fiables.

\section{«Les fatigués de la vie»}

C'est l'expression privilégiée qu'on entend aujourd'hui pour justifier que certains veulent en finir avec la vie. Il en va là d'un euphémisme fort douteux pour exprimer la banalisation du suicide. Après tout, n'avons-nous pas été un jour, vous et moi, des "fatigués de la vie»? Fatigués à cause d'une maladie qui n'était pas mortelle mais qui a fait beaucoup souffrir, fatigués à cause d'un burn-out difficile à vivre, à cause d'un divorce douloureusement traversé, d'une mise au chômage de longue durée ou de toute autre épreuve jugée à un moment donné insupportable? L'idée d'en finir nous a peut-être effleurés. Mais nous avons réussi à surmonter l'épreuve bien que le degré de souffrance fût parfois élevé. Pourquoi? Parce que nous avons en nous une faculté de résilience en vertu d'une foi en la vie plus forte que l'instinct de mort. Mais aussi et surtout parce nous avons trouvé auprès d'autrui une écoute attentive, une aide, un encouragement, une empathie active, une compassion qui nous a touchés précisément là où nous étions dans la souffrance, peut-être prêt à abandonner la partie, à en finir. Banaliser le suicide est une entreprise de destruction de la valeur la plus haute qui soit: notre vie elle-même!

De cet enjeu-là, qui est le foyer intime de notre existence personnelle et relationnelle, l'ASSM ne dit mot. Elle s'en tient souvent au caractère juridique du seul principe susceptible d'autoriser légalement la mort sans proposer de limites à la défense de la vie en jeu. L'Académie peut bien sûr se défendre de favoriser le suicide. Mais ce qu'elle ne voit pas, c'est que, si nous sommes des êtres évidemment vulnérables, nous sommes aussi des êtres de relation qui avons besoin des autres. C'est notre condition. La sociabilité est indispensable à notre état d'être humain vivant en société. L'isolement, la solitude sont le terreau fertile de l'enfermement sur soi et l'on sait que celui-ci est une des causes principales des tentatives de suicide. Non, il ne convient pas, comme le propose l'ASSM, de s'en référer à la seule détermination lucide du patient pour lui octroyer le droit de gagner sa mort assistée si facilement. Le patient en souffrance a besoin de tout autre chose: de retrouver le goût de vivre.

\section{Une sacralisation de l'autonomie}

Ce que l'ASSM ne voit pas et cautionne en fait par ses directives, c'est qu'on vit dans une société qui, depuis quelques décennies, a érigé l'individualisme en absolu. Chacun n'est responsable que de lui-même. Ce qui veut dire que chacun est finalement considéré isolément, livré en effet à sa seule référence subjective. Nul n'est plus responsable de la détresse des autres. Combien de gens - surtout des jeunes qui sont fragiles et manquent de repères qui leur donneraient des raisons de vivre combien de gens deviennent vulnérables et seuls. Ils sont parfois enfermés dans leur détresse en quête - au moins inconsciente - d'un salut souhaité. Va-t-on offrir à ces gens-là l'assistance au suicide parce qu'ils ressentent une souffrance jugée insupportable? Il faut savoir reconnaître que nous vivons dans une société qui a littéralement sacralisé l'autonomie de l'individu. Une société dominée par le relativisme, où le chacun pour soi n'est plus basé sur des valeurs reconnues comme porteuses de sens durable. Chacun est invité, poussé à consommer la vie sans entraves, sans plus considérer les liens sociaux comme fondés sur un minimum d'altruisme, de fraternité et de solidarité. Valeurs qui permettraient à ceux et celles qui sont seuls et sans repères de trouver des raisons de vivre plutôt que des motifs de se donner la mort.

Il ne faut pas s'étonner de la montée en puissance d'associations comme Exit ou Dignitas. Elles deviennent avec le temps des organisations actives de la mort programmée, cherchant et trouvant leur place dans les 
EMS où l'on pratique pourtant tout le contraire, c'està-dire des soins palliatifs dans le seul but d'accompagner et de soulager les souffrances des personnes en fin de vie. Par ses directives, l'ASSM va dans le même sens que ces associations.

\section{Etiam pueri! (Même les enfants!)}

Le point qui est le plus inadmissible dans la position de l'ASSM, c'est qu'elle n'hésite pas à stipuler que «les directives sont également applicables aux enfants et adolescents de tous âges» (ch. 1.1). Il est certes précisé «qu'il convient de vérifier au cas par cas la capacité de discernement par rapport à la décision à prendre». Le texte poursuit: «Si l'enfant ou l'adolescent est capable de discernement, le droit de décision passe des parents à l'enfant» (ch. 2.1). Voilà donc l'enfant seul, avec tout le poids de sa détermination momentanée, mais toujours révocable!

On côtoie ici l'intolérable. Car quel enfant ou adolescent de 12 à 16 ans est réellement capable de discernement à un âge largement reconnu comme fragile, fluctuant, instable et susceptible de retournement complet par rapport à sa propre décision momentanée de se donner la mort? Ce dont ces enfants et adolescents - soit-disant capables de discernement durable et sans véritable expérience de la vie - ont un urgent besoin, c'est d'une aide attentive pour les accompagner et leur donner des raisons de vivre. Des raisons de vivre et pas l'examen de leurs raisons de mourir! Ils sont à cet âge-là en pleine construction d'eux-mêmes, en recherche de sens et de confiance en la vie pour préparer leur avenir, un avenir qu'ils voudraient stable, valorisant. Là se trouve très concrètement le lieu où l'on voit combien notre société est malade.
Comment l'ASSM peut-elle soutenir l'application du suicide assisté aux enfants sinon parce qu'elle va jusqu'au bout d'une logique mortifère?

\section{Le rôle du médecin}

Qu'en est-il enfin du rôle du médecin selon l'ASSM? En 2018, l'ASSM reprend la même optique que celle proposée en 2004 et 2013: "L'aide au suicide ne fait pas partie de l'activité médicale car elle est contraire aux buts de la médecine.» Le texte actuel le précise: «Le rôle du médecin face à la fin de vie et à la mort consiste à soulager les symptômes et accompagner le patient. Il n'est de son devoir ni de proposer une assistance au suicide, ni de la pratiquer" (ch. 6.2.1). Mais que lit-on un peu plus loin? Ceci: «Si le patient persiste dans son désir (de suicide), le médecin peut, sur la base d'une décision dont il endosse personnellement la responsabilité, apporter une aide au suicide, sous réserve de cinq conditions» (dont les deux premières, les plus importantes, rappellent que «le patient doit être capable de discernement», puis que "son désir est mûrement réfléchi et ne résulte pas d'une pression extérieure»). Comment ne pas constater une contradiction entre l'affirmation selon laquelle l'aide au suicide est contraire aux buts de la médecine et l'affirmation opposée selon laquelle, dans la mesure où le patient veut vraiment se suicider, le médecin peut lui apporter une aide? C'est cautionner ainsi, quelles que soient les cinq conditions édictées par précaution, que le médecin est partie prenante du processus organisé par les associations d'aide au suicide comme Exit ou Dignitas. Le texte le reconnaît: "Chaque soignant doit décider lui-même si, pour lui, cette assistance est conforme aux objectifs de la médecine ou non» (ch. 6.2.1). Il sait très bien que non! 